\title{
New Pinning Synchronization of Complex Networks with Time-Varying Coupling Strength and Nondelayed and Delayed Coupling
}

\author{
Guoliang Wang, Zhongbao Yue, and Feng Wang \\ School of Information and Control Engineering, Liaoning Shihua University, Fushun, Liaoning 113001, China \\ Correspondence should be addressed to Guoliang Wang; gliangwang@aliyun.com
}

Received 23 September 2014; Revised 4 January 2015; Accepted 4 January 2015

Academic Editor: Hui Zhang

Copyright (c) 2015 Guoliang Wang et al. This is an open access article distributed under the Creative Commons Attribution License, which permits unrestricted use, distribution, and reproduction in any medium, provided the original work is properly cited.

\begin{abstract}
The pinning synchronization problem for a class of complex networks is studied by a stochastic viewpoint, in which both timevarying coupling strength and nondelayed and delayed coupling are included. Different from the traditionally similar methods, its interval is separated into two subintervals and described by a Bernoulli variable. Both bounds and switching probability of such subintervals are contained. Particularly, the nondelayed and delayed couplings occur alternately in which another independent Bernoulli variable is introduced. Then, a new kind of pinning controller without time-varying coupling strength signal is developed, in which only its bounds and probabilities are contained. When such probabilities are unavailable, two different kinds of adaption laws are established to make the complex network globally synchronous. Finally, the validity of the presented methods is proved through a numerical example.
\end{abstract}

\section{Introduction}

In the recent years, because of many systems in all fields of sciences and society, complex networks (CNs) have drawn a lot of attention. The property of CNs is that a large set of interconnected nodes are contained, and each node is an essential unit. Many natural and manmade systems can be found in our surroundings, such as ecosystems, internet, World Wide Web, social networks, and power grids, which are very important in our daily life. Due to the broad applications, the research of complex behaviors in complex networks has become a hot topic across many fields such as in [1-7].

In complex networks, such as ER random, small-world, and scale-free CNs [8-10], one of the most important collective behaviors is named as synchronization and has been studied extensively. Because of a large number of nodes existing in $\mathrm{CNs}$, it is unrealistic to control $\mathrm{CN}$ by adding controllers to all nodes. Then, it is natural to exploit a practical control strategy reducing the number of controllers usually referred to as pining control $[11,12]$. Up to now, a lot of results on pinning synchronization of various $\mathrm{CNs}$ have emerged, for example, [13-21]. When there are some parameters unknown, the problem of adaptive synchronization was reported in [2224]. If there are constant or time-varying delays in coupling, some delay-dependent criteria for synchronization stability were established in [25-27]. As for CN with nondelayed and delayed coupling, the pinning synchronization problem was considered in [28]. It is seen that the underlying system in this reference has the nondelayed and delayed couplings occurring simultaneously. If such two couplings happen separately with some probability, how to consider the pinning synchronization problem will be necessary and interesting. On the other hand, by investigating the aforementioned works, it is known that the coupling strength (CS) in most of them is constant. When CS is time-varying in a limited range, the global synchronization was realized by adjusting timevarying coupling strength (TCS) [29]. Based on the proposed methods, it is concluded that the adjusted CS will vary in a range, whose lower and upper bounds are finite. Though the given adjusting TCS is time-varying, its bounds used in realizing global synchronization may be different from the ones of the original TCS. Moreover, even if such bounds can be equal in theory, they may not be realized in practice. That is because the ability of instruments or actuators is limited 
in many practical applications. Thus, the method without considering the upper bound of CS may be conservative. In addition, the synchronization in [29] achieved by adjusting TCS needs every node state. It will make the realization not easy when CS has a great deal of nodes. Via exploiting the existing pinning algorithms, a state feedback controller will be constructed as one with TCS signal. In this case, the implementation of the designed pinning controller requires such a signal exactly available online. Unfortunately, from the point of application, this assumption is very ideal and hard to be satisfied. To the best of authors' knowledge, the pinning control problem of complex networks with TCS and nondelayed and delayed coupling has not yet been investigated, which motivates the current research.

In this paper, the pinning synchronization of complex networks with TCS and nondelayed and delayed coupling is studied by a stochastic viewpoint. Compared with the existing results of pinning control methods, the main contributions of this paper are as follows: (1) The pinning synchronization is realized by using a stochastic method. The original range of TCS is separated into two different subintervals, whose switching between such subintervals is described by a Bernoulli variable; (2) based on the proposed method, a kind of local state feedback controller is developed such that the resulting system is global synchronization in probability. It is seen that, without depending on TCS signal directly, the desired pinning controller is only related to the bounds and probabilities of such separated subintervals; (3) instead of the nondelayed and delayed couplings existing simultaneously, such couplings act on the underlying system asynchronously, which is modeled by another independent Bernoulli variable; (4) by the proposed results in this paper, it is concluded that such bounds and probabilities play important roles in the pinning synchronization, whose relationships are given and demonstrated in detail; (5) when such probabilities are unknown or inaccessible, two different adaptation laws for global synchronization are proposed, respectively.

Notations. $\mathbb{R}^{n}$ denotes the $n$ dimensional Euclidean space, $\mathbb{R}^{m \times n}$ is the set of all $m \times n$ real matrices. $\mathbb{C}\left([-\tau, 0], \mathbb{R}^{n}\right)$ is the Banach space of continuous functions mapping the interval $[\tau, 0]$ into $\mathbb{R}^{n}$ with the norm $|\phi|=\sup _{-\tau \leq \theta \leq 0}|\phi(\theta)|$, where $|x|$ denotes the usual Euclidean norm of a vector. $\mathscr{E}\{\cdot\}$ is the expectation operator with respect to some probability measure. $\lambda_{\max }(M)$ denote largest eigenvalues of a matrix $M$. $\mathscr{L}$ is the infinitesimal generator of $V(x, t)$ from $\mathbb{R}^{n} \times \mathbb{R}_{+}$to $\mathbb{R}^{n}$.

\section{Problem Formulation}

Consider a class of complex networks consisting of $N$ identical linearly and diffusively coupled nodes; it is described as

$$
\begin{aligned}
\dot{x}_{i}(t)= & f\left(x_{i}(t)\right)+\beta(t) c(t) \sum_{j=1}^{N} a_{i j} x_{j}(t) \\
& +(1-\beta(t)) c(t) \sum_{j=1}^{N} b_{i j} x_{j}(t-\tau), \quad i \in \mathbb{S},
\end{aligned}
$$

where $x_{i}(t)=\left(x_{i 1}(t), x_{i 2}(t), x_{i 3}(t), \ldots, x_{i n}(t)\right)^{T} \in \mathbb{R}^{n}, f$ : $\mathbb{R}^{n} \times \mathbb{R}_{+} \rightarrow \mathbb{R}^{n}$ is a differentiable function and is continuous, $\tau>0$ is the coupling delay, $c(t) \geq 0$ is the time-varying coupling strength and varies in $\Omega \triangleq\left[c_{1}, c_{2}\right]$ with $0 \leq c_{1} \leq$ $c_{2}<\infty$, and matrices $A \triangleq\left(a_{i j}\right)_{N \times N}$ and $B \triangleq\left(b_{i j}\right)_{N \times N}$ are the coupling configuration matrices and irreducible, whose elements $a_{i j}$ and $b_{i j}$ are described as follows: $a_{i j}=a_{j i} \geq 0$, for $i \neq j, a_{i j}=-\sum_{j=1, i \neq j}^{N} a_{i j}$, and $b_{i j}=b_{j i} \geq 0$, for $i \neq j$, and $b_{i j}=$ $-\sum_{j=1, i \neq j}^{N} b_{i j}, i, j \in \mathbb{S} \triangleq\{1,2, \ldots, N\}$. The initial conditions are given by $x_{i 0}(t)=\phi_{i}(t) \in \mathbb{C}\left([-\tau, 0], \mathbb{R}^{n}\right), \forall t \in[-\tau, 0] . \beta(t)$ is a Bernoulli variable and is described as follows:

$$
\beta(t)= \begin{cases}1, & \text { if nondelayed coupling acts, } \\ 0, & \text { if delayed coupling acts }\end{cases}
$$

whose probability is

$$
\begin{aligned}
& \mathbb{P}\{\beta(t)=1\}=\mathscr{E}\{\beta(t)\}=\beta^{*}, \\
& \mathbb{P}\{\beta(t)=0\}=1-\beta^{*} .
\end{aligned}
$$

In this paper, TCS $c(t)$ varies in $\left[c_{1}, c_{2}\right]$. That is, for any given constant $\widehat{c} \in\left[c_{1}, c_{2}\right], c(t)$ will take value in $\left[c_{1}, \widehat{c}\right]$ or $\left(\widehat{c}, c_{2}\right]$ randomly. Without loss of generality, $\widehat{c}$ can be chosen as $\widehat{c}=$ $(1 / 2)\left(c_{1}+c_{2}\right)$. So for any $c(t)$, two sets are described as

$$
\begin{aligned}
& \Omega_{1} \triangleq\left\{c(t): c(t) \in\left[c_{1}, \widehat{c}\right]\right\}, \\
& \Omega_{2} \triangleq\left\{c(t): c(t) \in\left(\widehat{c}, c_{2}\right]\right\} .
\end{aligned}
$$

A Bernoulli variable $\alpha(t)$ is described as

$$
\alpha(t)= \begin{cases}0, & c(t) \in \Omega_{1} \\ 1, & c(t) \in \Omega_{2}\end{cases}
$$

with

$$
\begin{aligned}
& \mathbb{P}\{\alpha(t)=1\}=\mathscr{E}\{\alpha(t)\}=\alpha^{*}, \\
& \mathbb{P}\{\alpha(t)=0\}=1-\alpha^{*},
\end{aligned}
$$

where $1-\alpha^{*}$ and $\alpha^{*}$ denote the probabilities of $c(t)$ taking values in $\Omega_{1}$ and $\Omega_{2}$, respectively. Moreover, it can be readily verified that

$$
\mathscr{E}\left\{\alpha(t)-\alpha^{*}\right\}=0 .
$$

Then, $c(t)$ is expressed as

$$
c(t)=(1-\alpha(t)) c_{1}(t)+\alpha(t) c_{2}(t),
$$

where $c_{1}(t)$ and $c_{2}(t)$ are described as

$$
\begin{aligned}
& c_{1}(t) \triangleq \begin{cases}c(t), & \text { if } \alpha(t)=0, \\
\widehat{c}, & \text { if } \alpha(t)=1,\end{cases} \\
& c_{2}(t) \triangleq \begin{cases}\widehat{c}, & \text { if } \alpha(t)=0, \\
c(t), & \text { if } \alpha(t)=1 .\end{cases}
\end{aligned}
$$


Then, system (1) is equivalent to

$$
\begin{aligned}
\dot{x}_{i}(t)= & f\left(x_{i}(t)\right)+\beta(t) \theta(t) \sum_{j=1}^{N} a_{i j} x_{j}(t) \\
& +(1-\beta(t)) \theta(t) \sum_{j=1}^{N} b_{i j} x_{j}(t-\tau), \quad i \in \mathbb{S},
\end{aligned}
$$

where

$$
\begin{aligned}
\theta(t) & =\theta_{1}(t)+\theta_{2}(t), \\
\theta_{1}(t) & =\left(\alpha(t)-\alpha^{*}\right)\left(c_{2}(t)-c_{1}(t)\right), \\
\theta_{2}(t) & =\left(1-\alpha^{*}\right) c_{1}(t)+\alpha^{*} c_{2}(t) .
\end{aligned}
$$

Let $s(t)$ be a solution to the isolated node of $\mathrm{CN}$; we have

$$
\dot{s}(t)=f(s(t))
$$

where $s(t)$ may be an equilibrium point, a nontrivial periodic orbit, or even a chaotic attractor. For complex network (10) with stochastic variable, some assumptions, definitions, and lemmas are needed to derive our main results.

Assumption 1 (see [15]). Supposing $P=\operatorname{diag}\left(p_{1}, p_{2}, \ldots, p_{n}\right)$ is a positive definite diagonal matrix and $\Delta=$ $\operatorname{diag}\left(\delta_{1}, \delta_{2}, \ldots, \delta_{n}\right)$ is a diagonal matrix, then, for some $\eta>0$, all $x, y \in \mathbb{R}^{n}$, and $t>0, f(x, t)$ can make the following inequality establish:

$$
\begin{aligned}
& (x-y)^{T} P(f(x)-f(y)-\Delta x+\Delta y) \\
& \leq-\eta(x-y)^{T}(x-y) .
\end{aligned}
$$

Definition 2. Complex network (5) is said to achieve globally asymptotical synchronization almost surely, for any initial conditions $x_{i 0}$ and $s_{0}$, if the following condition

$$
\mathbb{P}\left\{\lim _{t \rightarrow \infty}\left|x_{i}\left(t ; x_{i 0}\right)-s\left(t, s_{0}\right)\right|=0\right\}=1, \quad i \in \mathbb{S}
$$

holds.

Definition 3. The solution $x(t)$ to complex network (5) is said to be bounded almost surely if

$$
\sup _{t \geq 0}\left|x\left(t ; x_{0}\right)\right|<\infty \quad \text { a.s. }
$$

holds for all $x_{0} \in \mathbb{R}^{n N}$ with $x(t)=\left(x_{1}^{T}(t), x_{2}^{T}(t), \ldots, x_{N}^{T}(t)\right)^{T}$.

Lemma 4 (see [15]). Suppose $A \in \mathbb{R}^{N \times N}$ is in accordance with the following conditions:

(1) $a_{i j} \geq 0$, for $i \neq j$, and $a_{i j}=-\sum_{j=1, i \neq j}^{N} a_{i j}, \forall i, j \in \mathbb{S}$;

(2) A is irreducible; then one has the following:

(i) Real parts of the eigenvalues of $A$ are all negative, in addition to an eigenvalue 0 with the multiplicity 1 . (ii) $A$ has the right eigenvector $(1,1, \ldots, 1)^{T}$ corresponding to the eigenvalue 0 .

(iii) Let $\xi=\left(\xi_{1}, \xi_{2}, \ldots, \xi_{N}\right)^{T}$ be the left eigenvector of A corresponding to the eigenvalue 0; then one can let $\xi_{i}>0, i \in \mathbb{S}$.

Lemma 5. If $A=\left(a_{i j}\right)_{N \times N}$ is irreducible with $a_{i j}=a_{j i} \geq 0$ for $i \neq j$ and $\sum_{j=1}^{N} a_{i j}=0$ for $i \in \mathbb{S}$, then all the eigenvalues of the matrix

$$
\widetilde{A} \triangleq\left(\widetilde{a}_{i j}\right)_{N \times N}=A-K
$$

are negative, where $K \triangleq \operatorname{diag}\left\{k_{1}, k_{2}, \ldots, k_{N}\right\}$ with $k_{i}>0, i=$ $1,2, \ldots, l$, and $k_{i}=0, i \in \mathbb{S}_{l} \triangleq\{1,2, \ldots, l\}$.

Proof. Based on the result [12], it can be obtained directly. Thus it is omitted here.

If $A$ is an irreducible coupling matrix, let $\xi=$ $\left(\xi_{1}, \xi_{2}, \ldots, \xi_{N}\right)^{T}$ be the left eigenvector of $A$ corresponding to the eigenvalue 0 and with $\xi_{i}>0$, one has that matrix $\Xi=\operatorname{diag}\left(\xi_{1}, \xi_{2}, \ldots, \xi_{N}\right)$ is positive definite. If $A$ is irreducible, we have

$$
\begin{aligned}
\left(\Xi A+A^{T} \Xi\right)\left(\begin{array}{c}
1 \\
\vdots \\
1
\end{array}\right) & =\Xi A\left(\begin{array}{c}
1 \\
\vdots \\
1
\end{array}\right)+A^{T} \Xi\left(\begin{array}{c}
1 \\
\vdots \\
1
\end{array}\right) \\
& =\Xi A\left(\begin{array}{c}
1 \\
\vdots \\
1
\end{array}\right)+A^{T}\left(\begin{array}{c}
\xi_{1} \\
\vdots \\
\xi_{N}
\end{array}\right)=0
\end{aligned}
$$

which is irreducible. Letting $(\Xi \widetilde{A})^{s}=(1 / 2)\left(\Xi \widetilde{A}+\widetilde{A}^{T} \Xi\right)$, it is known from Lemma 5 that $(\Xi \widetilde{A})^{s}$ is negative definite.

Lemma 6. For any given appropriate matrices $\Omega, \Phi_{1}$, and $\Phi_{2}$ and scalars $\underline{\lambda}_{1} \leq \bar{\lambda}_{1}$ and $\underline{\lambda}_{2} \leq \bar{\lambda}_{2}$, then

$$
\Omega+\lambda_{1}(t) \Phi_{1}+\lambda_{2}(t) \Phi_{2}<0
$$

$$
\lambda_{1}(t) \in\left[\underline{\lambda}_{1}, \bar{\lambda}_{1}\right], \lambda_{2}(t) \in\left[\underline{\lambda}_{2}, \bar{\lambda}_{2}\right]
$$

if and only if

$$
\begin{aligned}
& \Omega+\underline{\lambda}_{1} \Phi_{1}+\underline{\lambda}_{2} \Phi_{2}<0, \\
& \Omega+\underline{\lambda}_{1} \Phi_{1}+\bar{\lambda}_{2} \Phi_{2}<0, \\
& \Omega+\bar{\lambda}_{1} \Phi_{1}+\underline{\lambda}_{2} \Phi_{2}<0, \\
& \Omega+\bar{\lambda}_{1} \Phi_{1}+\bar{\lambda}_{2} \Phi_{2}<0 .
\end{aligned}
$$

Proof. The necessity is obvious, since (18) holds. Next, we prove the sufficiency. From $\lambda_{1}(t) \in\left[\underline{\lambda}_{1}, \bar{\lambda}_{1}\right]$ and $\lambda_{2}(t) \epsilon$ $\left[\underline{\lambda}_{2}, \bar{\lambda}_{2}\right]$, it is concluded that

$$
\begin{array}{ll}
\lambda_{1}(t)=t_{1} \underline{\lambda}_{1}+\left(1-t_{1}\right) \bar{\lambda}_{1}, \quad \forall t_{1} \in[0,1], \\
\lambda_{2}(t)=t_{2} \underline{\lambda}_{2}+\left(1-t_{2}\right) \bar{\lambda}_{2}, \quad \forall t_{2} \in[0,1] .
\end{array}
$$


Then, from $(19) \times t_{1}$ plus $(21) \times\left(1-t_{1}\right)$, one gets

$$
\Omega+\underline{\lambda}_{1}(t) \Phi_{1}+\underline{\lambda}_{2} \Phi_{2} \leq 0
$$

Similarly,

$$
\Omega+\underline{\lambda}_{1}(t) \Phi_{1}+\bar{\lambda}_{2} \Phi_{2} \leq 0
$$

By applying similar method to (24) and (25), we obtain

$$
\Omega+\lambda_{1}(t) \Phi_{1}+\lambda_{2}(t) \Phi_{2} \leq 0
$$

where "=" is satisfied if and only if $t_{1}=0$ or $t_{1}=1$ and $t_{2}=$ 0 or $t_{2}=1$ hold simultaneously. However, without loss of generality, if $t_{1}=0$ and $t_{2}=0$, it is seen that

$$
\Omega+\bar{\lambda}_{1} \Phi_{1}+\bar{\lambda}_{2} \Phi_{2} \leq 0
$$

which is contradicted with (22). This completes the proof.

Here, the objective of pinning synchronization is to achieve $\mathbb{P}\left\{\lim _{t \rightarrow \infty}\left|x_{i}\left(t ; x_{i 0}\right)-s\left(t, s_{0}\right)\right|=0\right\}=1, i \in \mathbb{S}$, by using pinning control strategy on a small fraction $\delta(0<\delta \ll$ 1 ) of the total nodes in (5). Suppose that nodes $i_{1}, i_{2}, \ldots, i_{l}$ are selected, where $l=\lfloor\delta N\rfloor$ is the smaller, but a nearest integer to the real number $\delta N$. Without loss of generality, assume that the first $l$ nodes are selected to be pinned. A kind of local pinning controller without $c(t)$ but containing its bounds and probability distributions is designed as

$$
\begin{aligned}
\dot{x}_{i}(t)= & f\left(x_{i}(t)\right)+\beta(t) \theta(t) \sum_{j=1}^{N} a_{i j} x_{j}(t) \\
& +(1-\beta(t)) \theta(t) \sum_{j=1}^{N} b_{i j} x_{j}(t-\tau)+u_{i}(t), \\
\dot{x}_{i}(t)= & f\left(x_{i}(t)\right)+\beta(t) \theta(t) \sum_{j=1}^{N} a_{i j} x_{j}(t) \\
& +(1-\beta(t)) \theta(t) \sum_{j=1}^{N} b_{i j} x_{j}(t-\tau), \quad i \in \overline{\mathbb{S}},
\end{aligned}
$$

where

$$
\begin{aligned}
u_{i}(t) & =-\beta^{*} \widetilde{c} k_{i}\left(x_{i}(t)-s(t)\right), \quad k_{i}>0, \\
\widetilde{c} & =\left(1-\alpha^{*}\right) \widehat{c}+\alpha^{*} c_{2}, \\
\overline{\mathbb{S}} & =\mathbb{S}-\mathbb{S}_{l} .
\end{aligned}
$$

Letting $e_{i}(t)=x_{i}(t)-s(t)$, we have the error system as

$$
\begin{aligned}
\dot{e}_{i}(t)= & f\left(x_{i}(t)\right)-f(s(t)) \\
& +(1-\beta(t)) \theta(t) \sum_{j=1}^{N} b_{i j} x_{j}(t-\tau) \\
& +\beta(t) \theta(t) \sum_{j=1}^{N} a_{i j} x_{j}(t)-k e_{i}(t), \quad i \in \mathbb{S},
\end{aligned}
$$

where $k_{i}=0, i \in \overline{\mathbb{S}}$.
Remark 7. It is worth mentioning that, compared with some existing results such as $[5,11,18,28,29]$, complex network (28) in this paper is more general and has its properties. Firstly, the underlying system is more general in terms of having timevarying coupling strength, where nondelayed and delayed couplings take place synchronously. Secondly, a new kind of controller depending on its lower and upper bounds in addition to probabilities will be designed to realize the pinning synchronization, which is without TCS signal. However, in order to achieve this aim, new problems will be confronted, which will be mentioned below and should be dealt with.

\section{Main Results}

Theorem 8. Suppose Assumption 1 holds and A is a irreducible coupling matrix. If $k_{1}, k_{2}, \ldots, k_{l}$ are positive constants and

$$
\begin{aligned}
& 2 \delta_{j} \Xi+2 \beta^{*} \widetilde{c}(\Xi \widetilde{A})^{s}+I_{N}+\left(1-\beta^{*}\right)^{2} \widetilde{c}^{2} \lambda_{\max }^{2}(\Xi B) \\
& <0, \quad j \in \mathbb{T} \\
& 2 \delta_{j} \Xi+2 \beta^{*} \bar{c}(\Xi \widetilde{A})^{s}+I_{N}+\left(1-\beta^{*}\right)^{2} \bar{c}^{2} \lambda_{\max }^{2}(\Xi B) \\
& \quad<0, \quad j \in \mathbb{T},
\end{aligned}
$$

where $\lambda_{\max }(\Xi B)$ is the spectral radius of $\Xi B, \bar{c}=\left(1-\alpha^{*}\right) c_{1}+$ $\alpha^{*} \widehat{c}$, and $\mathbb{T} \triangleq\{1, \ldots, n\}$, complex network (28) achieves globally asymptotical synchronization almost surely.

Proof. The Lyapunov function is defined as

$$
\begin{aligned}
V(t)= & \frac{1}{2} \sum_{i=1}^{N} \xi_{i} e_{i}^{T}(t) P e_{i}(t) \\
& +\frac{1}{2} \sum_{i=1}^{n} p_{j} \int_{t-\tau}^{t} \tilde{e}_{j}^{T}(s) \widetilde{e}_{j}(s) d s,
\end{aligned}
$$

where $\xi_{i}>0$ is the element of the left eigenvector of $A$ corresponding the eigenvalue 0 , and $\widetilde{e}_{j}(t)=\left(e_{1 j}(t), e_{2 j}(t)\right.$, $\left.\ldots, e_{N j}(t)\right)^{T} \in \mathbb{R}^{N}, j \in \mathbb{T}$. Then we have

$$
\begin{aligned}
& \mathscr{L} V(t)=\sum_{i=1}^{N} \xi_{i} e_{i}^{T}(t) P\left[f\left(x_{i}(t)\right)-f(s(t))-\Delta e_{i}(t)\right] \\
& +\sum_{i=1}^{N} \xi_{i} e_{i}^{T} P \Delta e_{i}(t)+\sum_{i=1}^{N} \xi_{i} \theta_{2}(t) e_{i}^{T}(t) \\
& \cdot P\left[\beta^{*} \sum_{j=1}^{N} a_{i j} e_{j}(t)+\left(1-\beta^{*}\right) \sum_{j=1}^{N} b_{i j} e_{j}(t-\tau)\right] \\
& -\widetilde{c} \beta^{*} \sum_{q=1}^{l} \xi_{q} k_{q} e_{q}^{T}(t) P e_{q}(t)+\frac{1}{2} \\
& \cdot \sum_{j=1}^{n} p_{j}\left[\tilde{e}_{j}^{T}(t) \tilde{e}_{j}^{T}(t)-\tilde{e}_{j}^{T}(t-\tau) \tilde{e}_{j}^{T}(t-\tau)\right]
\end{aligned}
$$




$$
\begin{array}{lc}
\leq \sum_{i=1}^{N} \xi_{i} e_{i}^{T}(t) P\left[f\left(x_{i}(t)\right)-f(s(t))-\Delta e_{i}(t)\right] & \text { By Lemma 6, it is concluded that } Z_{j}(t)<0 \text { if } \\
+\sum_{i=1}^{N} \xi_{i} e_{i}^{T} P \Delta e_{i}(t)+\sum_{i=1}^{N} \xi_{i} \theta_{2}(t) e_{i}^{T}(t) & 2 \delta_{j} \Xi+2 \beta^{*} \widetilde{c}(\Xi \widetilde{A})^{s}+I_{N}+\left(1-\beta^{*}\right)^{2} \widetilde{c}^{2}(\Xi B)(\Xi B)^{T} \\
\cdot P\left[\beta^{*} \sum_{j=1}^{N} a_{i j} e_{j}(t)+\left(1-\beta^{*}\right) \sum_{j=1}^{N} b_{i j} e_{j}(t-\tau)\right] & <0, \quad j \in \mathbb{T}, \\
-\theta_{2}(t) \beta^{*} \sum_{q=1}^{l} \xi_{q} k_{q} e_{q}^{T}(t) P e_{q}(t)+\frac{1}{2} & 2 \delta_{j} \Xi+2 \beta^{*} \bar{c}(\Xi \widetilde{A})^{s}+I_{N}+\left(1-\beta^{*}\right)^{2} \bar{c}^{2}(\Xi B)(\Xi B)^{T} \\
& <0, \quad j \in \mathbb{T}
\end{array}
$$$$
\cdot \sum_{j=1}^{n} p_{j}\left[\tilde{e}_{j}^{T}(t) \tilde{e}_{j}^{T}(t)-\tilde{e}_{j}^{T}(t-\tau) \tilde{e}_{j}^{T}(t-\tau)\right]
$$$$
\leq-\eta \sum_{i=1}^{N} \xi_{i} e_{i}^{T}(t) e_{i}(t)+\sum_{j=1}^{n} p_{j} \delta_{j} \tilde{e}_{j}^{T}(t) \Xi \widetilde{e}_{j}(t)
$$$$
+\beta^{*} \theta_{2}(t) \sum_{j=1}^{n} p_{j} \widetilde{e}_{j}^{T}(t) \Xi \widetilde{A} \widetilde{e}_{j}^{T}(t)+\left(1-\beta^{*}\right) \theta_{2}(t)
$$$$
\cdot \sum_{j=1}^{n} p_{j} \tilde{e}_{j}^{T}(t) \Xi B \widetilde{e}_{j}^{T}(t-\tau)+\frac{1}{2}
$$$$
\cdot \sum_{j=1}^{n} p_{j}\left[\tilde{e}_{j}^{T}(t) \tilde{e}_{j}^{T}(t)-\tilde{e}_{j}^{T}(t-\tau) \tilde{e}_{j}^{T}(t-\tau)\right]
$$$$
\leq-\eta \sum_{i=1}^{N} \xi_{i} e_{i}^{T}(t) e_{i}(t)+\sum_{j=1}^{n} p_{j} \delta_{j} \tilde{e}_{j}^{T}(t) \Xi \widetilde{e}_{j}(t)
$$$$
+\beta^{*} \theta_{2}(t) \sum_{j=1}^{n} p_{j} \widetilde{e}_{j}^{T}(t)(\Xi \widetilde{A})^{s} \tilde{e}_{j}^{T}(t)+\left(1-\beta^{*}\right) \theta_{2}(t)
$$$$
\cdot \sum_{j=1}^{n} p_{j} \tilde{e}_{j}^{T}(t) \Xi B \widetilde{e}_{j}^{T}(t-\tau)+\frac{1}{2}
$$$$
\cdot \sum_{j=1}^{n} p_{j}\left[\tilde{e}_{j}^{T}(t) \tilde{e}_{j}^{T}(t)-\tilde{e}_{j}^{T}(t-\tau) \tilde{e}_{j}^{T}(t-\tau)\right]
$$$$
=-\eta \sum_{i=1}^{N} \xi_{i} e_{i}^{T}(t) e_{i}(t)+\frac{1}{2} \sum_{j=1}^{n} p_{j}\left(\widetilde{e}_{j}^{T}(t), \widetilde{e}_{j}^{T}(t-\tau)\right)
$$$$
\cdot Z_{j}(t)\left(\widetilde{e}_{j}(t), \widetilde{e}_{j}(t-\tau)\right)<0,
$$

where

$$
\begin{aligned}
& Z_{j}(t) \\
& =\left[\begin{array}{cc}
2 \delta_{j} \Xi+2 \beta^{*} \theta_{2}(t)(\Xi \widetilde{A})^{s}+I_{N} & \left(1-\beta^{*}\right) \theta_{2}(t) \Xi B \\
\left(1-\beta^{*}\right) \theta_{2}(t)(\Xi B)^{T} & -I_{N}
\end{array}\right],
\end{aligned}
$$

hold, which could be obtained by (31). Then, (33) holds. Thus, there is always a small scalar $\mu>0$ such that

$$
\begin{aligned}
\mathscr{E}\left\{V\left(e_{t}, t\right)\right\}-\mathscr{E}\left\{V\left(e_{0}, 0\right)\right\} \\
\leq-\mu \mathscr{E}\left\{\int_{0}^{t} e^{T}(s) e(s) d s\right\}
\end{aligned}
$$

which implies

$$
\mathscr{E}\left\{\int_{0}^{t} e^{T}(s) e(s) d s\right\} \leq \frac{1}{\mu} \mathscr{E}\left\{V\left(e_{0}, 0\right)\right\}<\infty
$$

By Dynkins formula, one gets

$$
\lim _{t \rightarrow \infty} \mathscr{E}\left\{|e(t)|^{2}\right\}=0
$$

which implies

$$
\mathbb{P}\left\{\lim _{t \rightarrow \infty}|e(t)|=0\right\}=1
$$

This completes the proof.

Remark 9. From Theorem 8, it is seen that both the lower bounds of the separated subintervals and the probabilities $\alpha^{*}$ and $\beta^{*}$ are involved. By using the existing methods dealing with TCS $c(t)$, it is known that only its lower bound is considered. Thus, for any given $K$, it is concluded that the lower bound $c_{1}$ obtained by Theorem 8 will be smaller than one obtained by the traditional methods only using its lower bound. In this sense, it is said that our result is less conservative. In other words, the range of $c(t)$ determined by (28) is usually larger than one obtained by considering $c_{1}$ only. Particularly, it is worth mentioning that such Bernoulli variables can also be replaced with Markov processes such as [30], since Bernoulli process is a special case of Markov process.

It is worth mentioning that the pinning controller in (28) needs $\alpha^{*}$ and $\beta^{*}$ to be known. When either of $\alpha^{*}$ and $\beta^{*}$ is unavailable, how to synchronize the complex network via using a pinning controller without $c(t)$ signal and to estimate such probabilities should be considered. In the following, adaptive pinning control laws will be developed to deal with them separately. 
Theorem 10. Suppose Assumption 1 holds and the coupling matrix $A$ is irreducible. If $k_{1}, \ldots, k_{l}$ are positive constants and

$$
\begin{aligned}
& 2 \delta_{j} \Xi+2 \beta^{*} c_{1}(\Xi \widetilde{A})^{s}+I_{N}+\left(1-\beta^{*}\right)^{2} c_{1}^{2} \lambda_{\max }^{2}(\Xi B) I_{N} \\
& \quad+c_{2} I_{N}<0, \\
& 2 \delta_{j} \Xi+2 \beta^{*} \widehat{c}(\Xi \widetilde{A})^{s}+I_{N}+\left(1-\beta^{*}\right)^{2} \widehat{c}^{2} \lambda_{\max }^{2}(\Xi B) I_{N} \\
& \quad+c_{2} I_{N}<0, \\
& \beta^{*}(\Xi \widetilde{A})^{s}+\frac{1}{4}\left(1-\beta^{*}\right)^{2} \lambda_{\max }^{2}(\Xi B) I_{N}<0
\end{aligned}
$$

hold for $\forall j \in \mathbb{T}$, complex network (28) achieves globally asymptotical synchronization almost surely under the adaptive pinning controller

$$
u_{i}(t)=-\beta^{*} \hat{c} k_{i} e_{i}(t)+v_{i}(t), \quad i \in \mathbb{S}_{l},
$$

where $v_{i}(t)=-\widehat{\alpha}(t) e_{i}(t)$, and the updating law

$$
\dot{\hat{\alpha}}(t)= \begin{cases}0, & \text { if } \hat{\alpha}(t)=1 \\ \rho \sum_{i=1}^{l} \xi_{i} e_{i}^{T}(t) P e_{i}(t), & \text { otherwise }\end{cases}
$$

with $\forall \rho>0$ and $\widehat{\alpha}_{0} \in[0,1]$.

Proof. Choose a Lyapunov function

$$
\begin{aligned}
V(t)= & \frac{1}{2} \sum_{i=1}^{N} \xi_{i} e_{i}^{T}(t) P e_{i}(t) \\
& +\frac{1}{2} \sum_{i=1}^{n} p_{j} \int_{t-\tau}^{t} \widetilde{e}_{j}^{T}(s) \widetilde{e}_{j}(s) d s+\frac{1}{2 \rho} \widetilde{\alpha}(t) \widetilde{\alpha}(t),
\end{aligned}
$$

where $\widetilde{\alpha}(t)=\widehat{\alpha}(t)-\alpha^{*}$. Then, for $\forall \zeta(t)=\left[e^{T}(t), \widetilde{\alpha}^{T}(t)\right]^{T} \neq 0$, we have

$$
\begin{aligned}
& \mathscr{L} V(t)=\sum_{i=1}^{N} \xi_{i} e_{i}^{T}(t) P\left[f\left(x_{i}(t)\right)-f(s(t))-\Delta e_{i}(t)\right] \\
& +\sum_{i=1}^{N} \xi_{i} e_{i}^{T}(t) P \Delta e_{i}(t)+\beta^{*} c_{1}(t) \sum_{i=1}^{N} \xi_{i} e_{i}^{T}(t) P \\
& \cdot \sum_{j=1}^{N} a_{i j} e_{j}(t)+\beta^{*} \widetilde{\theta}(t) \sum_{i=1}^{N} \xi_{i} e_{i}^{T}(t) P \sum_{j=1}^{N} a_{i j} e_{j}(t) \\
& +\left(1-\beta^{*}\right) c_{1}(t) \sum_{i=1}^{N} \xi_{i} e_{i}^{T}(t) P \sum_{j=1}^{N} b_{i j} e_{j}(t-\tau) \\
& +\frac{1}{\rho}\left(\widehat{\alpha}(t)-\alpha^{*}\right) \dot{\hat{\alpha}}(t)+\left(1-\beta^{*}\right) \widetilde{\theta}(t) \sum_{i=1}^{N} \xi_{i} e_{i}^{T}(t) P \\
& \cdot \sum_{j=1}^{N} b_{i j} e_{j}(t-\tau)-\beta^{*} \hat{c} \sum_{q=1}^{l} \xi_{q} k_{q} e_{q}^{T}(t) P e_{q}(t)+\frac{1}{2} \\
& \cdot \sum_{j=1}^{n} p_{j}\left[\widetilde{e}_{j}^{T}(t) \widetilde{e}_{j}(t)-\widetilde{e}_{j}^{T}(t-\tau) \widetilde{e}_{j}(t-\tau)\right]
\end{aligned}
$$

$+\sum_{i=1}^{N} \xi_{i} e_{i}^{T}(t) P v_{i}(t) \leq-\eta \sum_{i=1}^{N} \xi_{i} e_{i}^{T}(t) P e_{i}(t)$

$+\sum_{j=1}^{n} p_{j} \delta_{j} \tilde{e}_{j}^{T}(t) \Xi \widetilde{e}_{j}(t)+\beta^{*} c_{1}(t)$

$\cdot \sum_{j=1}^{n} p_{j} \widetilde{e}_{j}^{T}(t) \Xi \widetilde{A} \widetilde{e}_{j}(t)+\left(1-\beta^{*}\right) c_{1}(t)$

$\cdot \sum_{j=1}^{n} p_{j} \widetilde{e}_{j}^{T}(t) \Xi B \widetilde{e}_{j}(t-\tau)+\beta^{*} \widetilde{\theta}(t)$

$\cdot \sum_{j=1}^{n} p_{j} \widetilde{e}_{j}^{T}(t) \Xi \widetilde{A} \widetilde{e}_{j}(t)+\left(1-\beta^{*}\right) \widetilde{\theta}(t)$

$\cdot \sum_{j=1}^{n} p_{j} \widetilde{e}_{j}^{T}(t) \Xi B \widetilde{e}_{j}(t-\tau)+\sum_{i=1}^{N} \xi_{i} e_{i}^{T}(t) P v_{i}(t)+\frac{1}{2}$

$\cdot \sum_{j=1}^{n} p_{j}\left[\tilde{e}_{j}^{T}(t) \tilde{e}_{j}(t)-\tilde{e}_{j}^{T}(t-\tau) \tilde{e}_{j}(t-\tau)\right]$

$+\frac{1}{\rho}\left(\widehat{\alpha}(t)-\alpha^{*}\right) \dot{\hat{\alpha}}(t) \leq-\eta \sum_{i=1}^{N} \xi_{i} e_{i}^{T}(t) P e_{i}(t)+\beta^{*}$

$+\sum_{j=1}^{n} p_{j} \delta_{j} \widetilde{e}_{j}^{T}(t) \Xi \widetilde{e}_{j}(t)+\beta^{*} c_{1}(t)$

$\cdot \sum_{j=1}^{n} p_{j} \tilde{e}_{j}^{T}(t)(\Xi \widetilde{A})^{s} \widetilde{e}_{j}(t)+\left(1-\beta^{*}\right) c_{1}(t)$

$\cdot \sum_{j=1}^{n} p_{j} \widetilde{e}_{j}^{T}(t) \Xi B \widetilde{e}_{j}(t-\tau) \widetilde{\theta}(t)$

$\cdot \sum_{j=1}^{n} p_{j} \widetilde{e}_{j}^{T}(t)(\Xi \widetilde{A})^{s} \widetilde{e}_{j}(t)+\left(1-\beta^{*}\right) \widetilde{\theta}(t)$

$\cdot \sum_{j=1}^{n} p_{j} \widetilde{e}_{j}^{T}(t) \Xi B \widetilde{e}_{j}(t-\tau)+\sum_{i=1}^{N} \xi_{i} e_{i}^{T}(t) P v_{i}(t)+\frac{1}{2}$

$\cdot \sum_{j=1}^{n} p_{j}\left[\tilde{e}_{j}^{T}(t) \tilde{e}_{j}(t)-\tilde{e}_{j}^{T}(t-\tau) \tilde{e}_{j}(t-\tau)\right]$

$+\frac{1}{\rho}\left(\widehat{\alpha}(t)-\alpha^{*}\right) \dot{\hat{\alpha}}(t)=-\eta \sum_{i=1}^{N} \xi_{i} e_{i}^{T}(t) P e_{i}(t)$

$+\sum_{j=1}^{n} p_{j} \tilde{e}_{j}^{T}(t)\left[\delta_{j} \Xi+\beta^{*} c_{1}(t)(\Xi \widetilde{A})^{s}+\frac{1}{2} I_{N}\right] \widetilde{e}_{j}(t)$

$+\left(1-\beta^{*}\right) c_{1}(t) \sum_{j=1}^{n} p_{j} \widetilde{e}_{j}^{T}(t) \Xi B \widetilde{e}_{j}(t-\tau)-\frac{1}{2}$

$\cdot \sum_{j=1}^{n} p_{j} \tilde{e}_{i}^{T}(t-\tau) \widetilde{e}_{i}(t-\tau)+\beta^{*} \widetilde{\theta}(t)$ 


$$
\begin{aligned}
& \cdot \sum_{j=1}^{n} p_{j} \tilde{e}_{j}^{T}(t)(\Xi \widetilde{A})^{s} \widetilde{e}_{j}(t)+\left(1-\beta^{*}\right) \tilde{\theta}(t) \\
& \cdot \sum_{j=1}^{n} p_{j} \widetilde{e}_{j}^{T}(t) \Xi B \widetilde{e}_{j}(t-\tau)+\sum_{i=1}^{N} \xi_{i} e_{i}^{T}(t) P v_{i}(t) \\
& +\frac{1}{\rho}\left(\widehat{\alpha}(t)-\alpha^{*}\right) \dot{\hat{\alpha}}(t)-\widetilde{\theta}(t) \\
& \cdot \sum_{i=1}^{n} p_{j} \tilde{e}_{j}^{T}(t-\tau) \tilde{e}_{j}(t-\tau)+\tilde{\theta}(t) \\
& \cdot \sum_{i=1}^{n} p_{j} \tilde{e}_{j}^{T}(t-\tau) \tilde{e}_{j}(t-\tau) \leq-\eta \sum_{i=1}^{N} \xi_{i} e_{i}^{T}(t) P e_{i}(t) \\
& +\sum_{j=1}^{n} p_{j} \widetilde{e}_{j}^{T}(t)\left[\delta_{j} \Xi+\beta^{*} c_{1}(t)(\Xi \widetilde{A})^{s}+\frac{1}{2} I_{N}\right] \widetilde{e}_{j}(t) \\
& +\left(1-\beta^{*}\right) c_{1}(t) \sum_{j=1}^{n} p_{j} \widetilde{e}_{j}^{T}(t) \Xi B \widetilde{e}_{j}(t-\tau)-\frac{1}{2} \\
& \text { where } \\
& Z_{j}(t) \\
& =\left[\begin{array}{cc}
2 \delta_{j} \Xi+2 \beta^{*} c_{1}(t)(\Xi \widetilde{A})^{s}+I_{N}+c_{2} I_{N} & \left(1-\beta^{*}\right) c_{1}(t) \Xi B \\
\left(1-\beta^{*}\right) c_{1}(\Xi B)^{T} & -I_{N}
\end{array}\right], \\
& M_{j}=\left[\begin{array}{cc}
\beta^{*}(\Xi \widetilde{A})^{s} & \frac{1}{2}\left(1-\beta^{*}\right) \Xi B \\
\frac{1}{2}\left(1-\beta^{*}\right)(\Xi B)^{T} & -I_{N}
\end{array}\right], \quad j \in \mathbb{T} \\
& \tilde{\theta}(t)=\alpha^{*}\left(c_{2}(t)-c_{1}(t)\right) \text {. } \\
& 2 \delta_{j} \Xi+2 \beta^{*} c_{1}(\Xi \widetilde{A})^{s}+I_{N}+\left(1-\beta^{*}\right)^{2} c_{1}^{2}(\Xi B)(\Xi B)^{T} \\
& +c_{2} I_{N}<0 \text {, } \\
& 2 \delta_{j} \Xi+2 \beta^{*} \widehat{c}(\Xi \widetilde{A})^{s}+I_{N}+\left(1-\beta^{*}\right)^{2} \widehat{c}^{2}(\Xi B)(\Xi B)^{T} \\
& +c_{2} I_{N}<0
\end{aligned}
$$$$
\cdot \sum_{j=1}^{n} p_{j} \tilde{e}_{j}^{T}(t-\tau) \widetilde{e}_{j}(t-\tau)+\beta^{*} \widetilde{\theta}(t)
$$$$
\cdot \sum_{j=1}^{n} p_{j} \tilde{e}_{j}^{T}(t)(\Xi \widetilde{A})^{s} \widetilde{e}_{j}(t)+\left(1-\beta^{*}\right) \widetilde{\theta}(t)
$$$$
\cdot \sum_{j=1}^{n} p_{j} \widetilde{e}_{j}^{T}(t) \Xi B \widetilde{e}_{j}(t-\tau)+\sum_{i=1}^{N} \xi_{i} e_{i}^{T}(t) P v_{i}(t)
$$$$
+\frac{1}{\rho}\left(\widehat{\alpha}(t)-\alpha^{*}\right) \dot{\hat{\alpha}}(t)-\tilde{\theta}(t)
$$$$
\cdot \sum_{i=1}^{n} p_{j} \widetilde{e}_{j}^{T}(t-\tau) \widetilde{e}_{j}(t-\tau)
$$$$
+c_{2} \sum_{i=1}^{n} p_{j} \widetilde{e}_{j}^{T}(t-\tau) \widetilde{e}_{j}(t-\tau)=-\left(\eta+\alpha^{*}\right)
$$$$
\cdot \sum_{i=1}^{N} \xi_{i} e_{i}^{T}(t) P e_{i}(t)+\frac{1}{2}
$$$$
\cdot \sum_{j=1}^{n} p_{j}\left(\widetilde{e}_{j}^{T}(t), \widetilde{e}_{j}^{T}(t-\tau)\right) Z_{j}(t)\left(\widetilde{e}_{j}(t), \widetilde{e}_{j}(t-\tau)\right)
$$$$
+\widetilde{\theta}(t)
$$$$
\cdot \sum_{j=1}^{n} p_{j}\left(\tilde{e}_{j}^{T}(t), \tilde{e}_{j}^{T}(t-\tau)\right) M_{j}\left(\widetilde{e}_{j}(t), \widetilde{e}_{j}(t-\tau)\right)
$$$$
\leq 0
$$

hold for $j \in \mathbb{S}$. Similarly, $M_{j}<0$ is equivalent to

$$
\beta^{*}(\Xi \widetilde{A})^{s}+\frac{1}{4}\left(1-\beta^{*}\right)^{2}(\Xi B)(\Xi B)^{T}<0 .
$$

From conditions (31)-(32), it is obtained that (46)-(47) hold. Then, one has (44) which implies

$$
\mathscr{E}\{V(\zeta(t), t)\} \leq \mathscr{E}\left\{V\left(\zeta_{0}, 0\right)\right\} .
$$

For any $t \geq 0, k>0$, when $\sup _{0 \leq s \leq t}|\zeta(s)| \geq k$, and from (48), one obtains

$$
\begin{gathered}
\mathbb{P}\left\{\sup _{0 \leq s \leq t}|\zeta(s)| \geq k\right\} \inf _{|\zeta| \geq k, t \geq 0} V(\zeta(t), t) \\
\leq \mathscr{E}\{V(\zeta(t), t)\} \leq \mathscr{E}\left\{V\left(\zeta_{0}, 0\right)\right\}
\end{gathered}
$$

which implies

$$
\mathbb{P}\left\{\sup _{0 \leq s \leq t}|\zeta(s)| \geq k\right\} \leq \frac{\mathscr{E}\left\{V\left(\zeta_{0}, 0\right)\right\}}{\inf _{|\zeta| \geq k, t \geq 0} V(\zeta(t), t)} .
$$

From (43), it is concluded that $\inf _{|\zeta| \geq k, t \geq 0} V(\zeta(t), t) \rightarrow \infty$, as $k \rightarrow \infty$. Based on this and taking into account (50), one concludes that $\zeta(t)$ is bounded almost surely. On the other hand, because of (44), there is $\mu>0$ such that

$$
\begin{aligned}
\mathscr{E}\{V(\zeta(t), t)\}-\mathscr{E}\left\{V\left(\zeta_{0}, 0\right)\right\} \\
\leq-\mu \mathscr{E}\left\{\int_{0}^{t} e^{T}(s) e(s) d s\right\} .
\end{aligned}
$$

Then, it is directly obtained that

$$
\mathscr{E}\left\{\int_{0}^{t} e^{T}(s) e(s) d s\right\} \leq \frac{1}{\mu} \mathscr{E}\left\{V\left(\zeta_{0}, 0\right)\right\}<\infty .
$$

Similar to the proof of (37), it is concluded that complex network (28) plus adaptive pinning controller (41) is asymptotical synchronization almost surely and the unknown probability $\alpha^{*}$ is estimated. This completes the proof. 
Remark 11. Compared with some existing references, the adaptive pinning controller (33) has its properties which are listed as follows. Firstly, the adaptive controller is used to estimate a scalar in a range and having an upper finite bound, while the other results such as $[20,22,28]$ are used to estimate control gains and without this restriction. Secondly, when $c(t)$ varies in a range, a new class of pinning controller in this paper is designed without signal $c(t)$, which is different from that in [29]. Moreover, instead of $c(t)$ without an upper bound, it is more practical that it is in a finite range. Thirdly, some adaption controllers such as in $[17,22,24]$ should be added to all the nodes of complex network. It is impossible to realize these controllers for complex network with large nodes. In addition, the adaption controller in [17] can cause chattering as the error state can reach the discontinuity region before the complex network achieves the global synchronization. However, this is not true for adaption controller (33) which is also on a small fraction of nodes in complex networks.

Theorem 12. Suppose Assumption 1 holds and the coupling matrix $A$ is irreducible. If $k_{1}, \ldots, k_{l}$ are positive constants and satisfy

$$
\begin{gathered}
2 \delta_{j} \Xi+(1-2 \widetilde{c}) I_{N}+\widetilde{c}^{2} \lambda_{\max }^{2}(\Xi B) I_{N}<0, \quad j \in \mathbb{T} \\
2 \delta_{j} \Xi+(1-2 \widetilde{c}) I_{N}+\bar{c}^{2} \lambda_{\max }^{2}(\Xi B) I_{N}<0, \quad j \in \mathbb{T} \\
(\Xi \widetilde{A})^{s}+\frac{1}{4}(\Xi B)(\Xi B)^{T}<0
\end{gathered}
$$

the complex network achieves globally asymptotical synchronization almost surely under the adaptive pinning controller

$$
\begin{array}{ll}
u_{i}(t)=-\widetilde{c} k_{i} e_{i}(t)+v_{i}(t), \quad i \in \mathbb{S}_{l}, \\
v_{i}(t)= \begin{cases}0, & \text { if } \sum_{i=1}^{l}\left|e_{i}(t)\right|^{2}=0, \\
-\frac{P^{-1} e_{i}(t)}{\sum_{i=1}^{l} \xi_{i}\left|e_{i}(t)\right|^{2}} \widetilde{c}[\Phi(e(t))+\widehat{\beta}(t) \Phi(e(t-\tau))], & \text { otherwise }\end{cases}
\end{array}
$$

with $\Phi(e(t))=\sum_{i=1}^{N} e_{i}^{T}(t) P e_{i}(t)$ and the updating laws

$$
\dot{\hat{\beta}}(t)= \begin{cases}0, & \text { if } \widehat{\beta}(t)=1 \\ \rho \widetilde{c} \sum_{i=1}^{N} e_{i}^{T}(t-\tau) P e_{i}(t-\tau), & \text { otherwise }\end{cases}
$$

with $\forall \rho>0$ and $\widehat{\beta}_{0} \in[0,1]$.

Proof. Define the Lyapunov function as

$$
\begin{aligned}
V(t)= & \frac{1}{2} \sum_{i=1}^{N} \xi_{i} e_{i}^{T}(t) P e_{i}(t) \\
& +\frac{1}{2} \sum_{i=1}^{n} p_{j} \int_{t-\tau}^{t} \tilde{e}_{j}^{T}(s) \widetilde{e}_{j}(s) d s+\frac{1}{2 \rho} \widetilde{\beta}(t) \widetilde{\beta}(t),
\end{aligned}
$$

where $\xi_{i}$ is the element of the left eigenvector of $A$ corresponding to the eigenvalue 0 and $\widetilde{\beta}(t)=\widehat{\beta}(t)-\beta^{*}$. Then, for $\forall \zeta(t)=\left[e^{T}(t), \tilde{\alpha}^{T}(t)\right]^{T} \neq 0$, we have

$$
\begin{gathered}
\mathscr{L} V(t)=\sum_{i=1}^{N} \xi_{i} e_{i}^{T}(t) P\left[f\left(x_{i}(t)\right)-f(s(t))-\Delta e_{i}(t)\right] \\
+\sum_{i=1}^{N} \xi_{i} e_{i}^{T}(t) P \Delta e_{i}(t)+\beta^{*} \theta_{2}(t) \sum_{i=1}^{N} \xi_{i} e_{i}^{T}(t) P \\
. \sum_{j=1}^{N} a_{i j} e_{j}(t)+\left(1-\beta^{*}\right) \theta_{2}(t) \sum_{i=1}^{N} \xi_{i} e_{i}^{T}(t) P
\end{gathered}
$$

$$
\begin{aligned}
& \cdot \sum_{j=1}^{N} b_{i j} e_{j}(t-\tau)-\tilde{c} \sum_{q=1}^{l} \xi_{q} k_{q} e_{q}^{T}(t) P e_{q}(t)+\frac{1}{2} \\
& \cdot \sum_{j=1}^{n} p_{j}\left[\tilde{e}_{j}^{T}(t) \tilde{e}_{j}(t)-\tilde{e}_{j}^{T}(t-\tau) \tilde{e}_{j}(t-\tau)\right] \\
& +\frac{1}{\rho}\left(\widehat{\beta}(t)-\beta^{*}\right) \dot{\hat{\beta}}(t)+\sum_{i=1}^{N} \xi_{i} e_{i}^{T}(t) P v_{i}(t) \\
& \leq \sum_{i=1}^{N} \xi_{i} e_{i}^{T}(t) P\left[f\left(x_{i}(t)\right)-f(s(t))-\Delta e_{i}(t)\right] \\
& +\sum_{i=1}^{N} \xi_{i} e_{i}^{T}(t) P \Delta e_{i}(t)+\beta^{*} \theta_{2}(t) \sum_{i=1}^{N} \xi_{i} e_{i}^{T}(t) P \\
& \cdot \sum_{j=1}^{N} a_{i j} e_{j}(t)+\left(1-\beta^{*}\right) \theta_{2}(t) \sum_{i=1}^{N} \xi_{i} e_{i}^{T}(t) P \\
& \cdot \sum_{j=1}^{N} b_{i j} e_{j}(t-\tau)-\beta^{*} \theta_{2}(t) \sum_{q=1}^{l} \xi_{q} k_{q} e_{q}^{T}(t) P e_{q}(t)+\frac{1}{2} \\
& \cdot \sum_{j=1}^{n} p_{j}\left[\tilde{e}_{j}^{T}(t) \widetilde{e}_{j}(t)-\tilde{e}_{j}^{T}(t-\tau) \widetilde{e}_{j}(t-\tau)\right] \\
& +\frac{1}{\rho}\left(\widehat{\beta}(t)-\beta^{*}\right) \dot{\hat{\beta}}(t)+\sum_{i=1}^{N} \xi_{i} e_{i}^{T}(t) P v_{i}(t)
\end{aligned}
$$




$$
\begin{aligned}
& \leq-\eta \sum_{i=1}^{N} \xi_{i} e_{i}^{T}(t) P e_{i}(t)+\sum_{j=1}^{n} p_{j} \delta_{j} \widetilde{e}_{j}^{T}(t) \Xi \widetilde{e}_{j}(t) \\
& +\beta^{*} \theta_{2}(t) \sum_{j=1}^{n} p_{j} \widetilde{e}_{j}^{T}(t) \Xi \widetilde{A} \widetilde{e}_{j}(t)+\left(1-\beta^{*}\right) \theta_{2}(t) \\
& \cdot \sum_{j=1}^{n} p_{j} \widetilde{e}_{j}^{T}(t) \Xi B \widetilde{e}_{j}(t-\tau)+\frac{1}{2} \\
& \cdot \sum_{j=1}^{n} p_{j}\left[\tilde{e}_{j}^{T}(t) \widetilde{e}_{j}(t)-\tilde{e}_{j}^{T}(t-\tau) \widetilde{e}_{j}(t-\tau)\right] \\
& +\frac{1}{\rho}\left(\widehat{\beta}(t)-\beta^{*}\right) \dot{\hat{\beta}}(t)+\sum_{i=1}^{N} \xi_{i} e_{i}^{T}(t) P v_{i}(t) \\
& =-\eta \sum_{i=1}^{N} \xi_{i} e_{i}^{T}(t) P e_{i}(t)+\beta^{*} \theta_{2}(t) \\
& \cdot\left[\sum_{j=1}^{n} p_{j} \widetilde{e}_{j}^{T}(t) \Xi \widetilde{A} \widetilde{e}_{j}(t)-\sum_{j=1}^{n} p_{j} \widetilde{e}_{j}^{T}(t) \Xi B \widetilde{e}_{j}(t-\tau)\right] \\
& +\sum_{j=1}^{n} p_{j} \tilde{e}_{j}^{T}(t)\left(\delta_{j} \Xi+\frac{1}{2} I_{N}\right) \widetilde{e}_{j}(t)+\theta_{2}(t) \\
& \cdot \sum_{j=1}^{n} p_{j} \widetilde{e}_{j}^{T}(t) \Xi B \widetilde{e}_{j}(t-\tau)-\frac{1}{2} \\
& \cdot \sum_{j=1}^{n} p_{j} \tilde{e}_{j}^{T}(t-\tau) \widetilde{e}_{j}(t-\tau)+\frac{1}{\rho}\left(\widehat{\beta}(t)-\beta^{*}\right) \dot{\hat{\beta}}(t) \\
& +\sum_{i=1}^{N} \xi_{i} e_{i}^{T}(t) P v_{i}(t)=-\eta \sum_{i=1}^{N} \xi_{i} e_{i}^{T}(t) P e_{i}(t)+\frac{1}{2} \\
& \cdot \sum_{j=1}^{n} p_{j}\left(\widetilde{e}_{j}^{T}(t), \widetilde{e}_{j}^{T}(t-\tau)\right) \bar{Z}_{j}(t)\left(\widetilde{e}_{j}(t), \widetilde{e}_{j}(t-\tau)\right) \\
& +\beta^{*} \theta_{2}(t) \\
& \cdot\left[\sum_{j=1}^{n} p_{j} \widetilde{e}_{j}^{T}(t) \Xi \widetilde{A} \widetilde{e}_{j}(t)-\sum_{j=1}^{n} p_{j} \widetilde{e}_{j}^{T}(t) \Xi B \widetilde{e}_{j}(t-\tau)\right]
\end{aligned}
$$

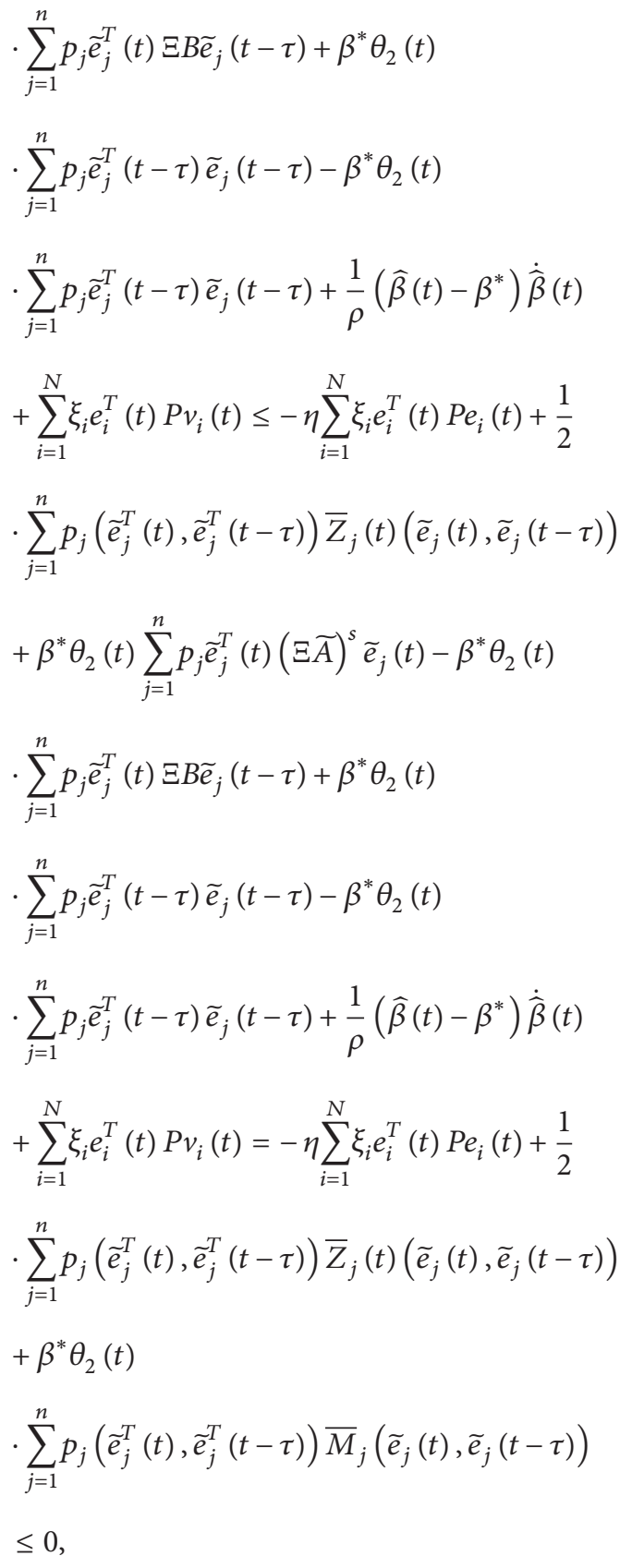$$
+\frac{1}{\rho}\left(\widehat{\beta}(t)-\beta^{*}\right) \dot{\hat{\beta}}(t)+\sum_{i=1}^{N} \xi_{i} e_{i}^{T}(t) P v_{i}(t)
$$$$
=-\eta \sum_{i=1}^{N} \xi_{i} e_{i}^{T}(t) P e_{i}(t)+\frac{1}{2}
$$$$
\cdot \sum_{j=1}^{n} p_{j}\left(\tilde{e}_{j}^{T}(t), \tilde{e}_{j}^{T}(t-\tau)\right) \bar{Z}_{j}(t)\left(\tilde{e}_{j}(t), \tilde{e}_{j}(t-\tau)\right)
$$$$
+\beta^{*} \theta_{2}(t) \sum_{j=1}^{n} p_{j} \tilde{e}_{j}^{T}(t) \Xi \widetilde{A} \widetilde{e}_{j}(t)-\beta^{*} \theta_{2}(t)
$$

where

$$
\begin{aligned}
\bar{Z}_{j}(t) & =\left[\begin{array}{cc}
2 \delta_{j} \Xi+(1-2 \widetilde{c}) I_{N} & \theta_{2}(t) \Xi B \\
\theta_{2}(t)(\Xi B)^{T} & -I_{N}
\end{array}\right], \quad j \in \mathbb{T}, \\
\bar{M}_{j} & =\left[\begin{array}{cc}
(\Xi \widetilde{A})^{s} & -\frac{1}{2} \Xi B \\
-\frac{1}{2}(\Xi B)^{T} & -I_{N}
\end{array}\right], \quad j \in \mathbb{T} .
\end{aligned}
$$

Similarly, one has that $\bar{Z}_{j}(t)<0$ is guaranteed by

$$
\begin{aligned}
& 2 \delta_{j} \Xi+(1-2 \widetilde{c}) I_{N}+\widetilde{c}^{2}(\Xi B)(\Xi B)^{T}<0, \quad j \in \mathbb{T}, \\
& 2 \delta_{j} \Xi+(1-2 \widetilde{c}) I_{N}+\bar{c}^{2}(\Xi B)(\Xi B)^{T}<0, \quad j \in \mathbb{T},
\end{aligned}
$$


where $\bar{M}_{j}<0$ is obtained by

$$
(\Xi \widetilde{A})^{s}+\frac{1}{4}(\Xi B)(\Xi B)^{T}<0 .
$$

Based on (53), we have (59)-(60). The next is similar to the proof of Theorem 10. This completes the proof.

\section{Numerical Examples}

In this section, a simulation example is used to illustrate the usefulness of our main results.

Example 1. Consider that a dynamical node of complex network is a Lorenz system. It is described as

$$
\begin{aligned}
& \dot{x}_{1}(t)=a\left(x_{2}(t)-x_{1}(t)\right), \\
& \dot{x}_{2}(t)=c x_{1}(t)-x_{1}(t) x_{x}(t)-x_{2}(t), \\
& \dot{x}_{3}(t)=x_{1}(t) x_{2}(t)-b x_{3}(t) .
\end{aligned}
$$

When the parameters are given as $a=10, b=8 / 3$, and $c=$ 28, Lorenz system has a chaotic attractor shown in Figure 1. We take $P=\operatorname{diag}\{24,0.002,12\}$ and $\Delta=\operatorname{diag}\{3,3,3\}$ such that Lorenz system (61) satisfies Assumption 1. Suppose an undirected $\mathrm{E}-\mathrm{R}$ random network consisting of $N=10$ nodes with $p=0.8$, where the coupling matrix is

A

$$
=\left(\begin{array}{cccccccccc}
-6 & 1 & 0 & 0 & 1 & 0 & 1 & 1 & 1 & 1 \\
1 & -6 & 0 & 1 & 1 & 1 & 0 & 0 & 1 & 1 \\
0 & 0 & -6 & 1 & 1 & 1 & 1 & 1 & 1 & 0 \\
0 & 1 & 1 & -7 & 1 & 0 & 1 & 1 & 1 & 1 \\
1 & 1 & 1 & 1 & -7 & 0 & 1 & 1 & 0 & 1 \\
0 & 1 & 1 & 0 & 0 & -6 & 1 & 1 & 1 & 1 \\
1 & 0 & 1 & 1 & 1 & 1 & -7 & 1 & 0 & 1 \\
1 & 0 & 1 & 1 & 1 & 1 & 1 & -6 & 0 & 0 \\
1 & 1 & 1 & 1 & 0 & 1 & 0 & 0 & -5 & 0 \\
1 & 1 & 0 & 1 & 1 & 1 & 1 & 0 & 0 & -6
\end{array}\right),
$$

$B$

$$
=\left(\begin{array}{cccccccccc}
-5 & 1 & 0 & 1 & 0 & 0 & 1 & 0 & 1 & 1 \\
1 & -5 & 0 & 0 & 1 & 1 & 0 & 0 & 1 & 1 \\
0 & 0 & -4 & 1 & 1 & 0 & 0 & 1 & 1 & 0 \\
1 & 0 & 1 & -5 & 0 & 1 & 1 & 0 & 0 & 1 \\
0 & 1 & 1 & 0 & -4 & 0 & 0 & 1 & 0 & 1 \\
0 & 1 & 0 & 1 & 0 & -5 & 1 & 0 & 1 & 1 \\
1 & 0 & 0 & 1 & 0 & 1 & -3 & 0 & 0 & 0 \\
0 & 0 & 1 & 0 & 1 & 0 & 0 & -3 & 0 & 1 \\
1 & 1 & 1 & 0 & 0 & 1 & 0 & 0 & -4 & 0 \\
1 & 1 & 0 & 1 & 1 & 1 & 0 & 1 & 0 & -6
\end{array}\right) .
$$

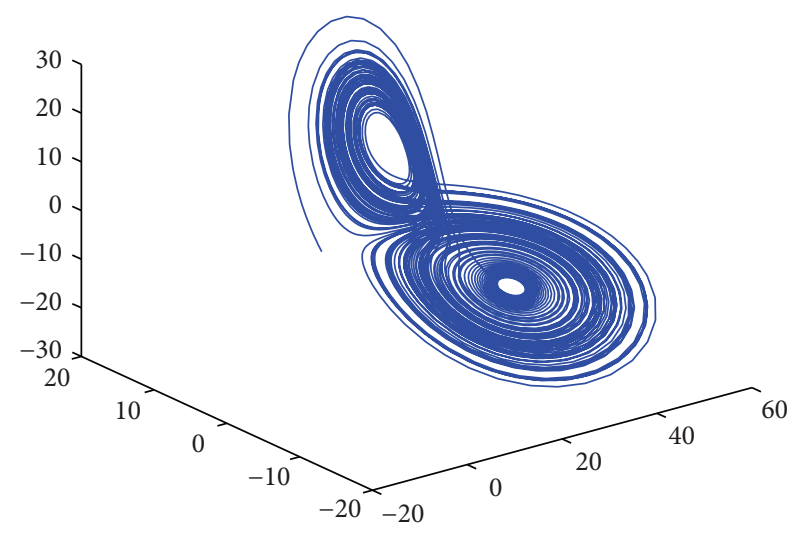

FIGURE 1: Chaotic orbit of Lorenz's circuit.

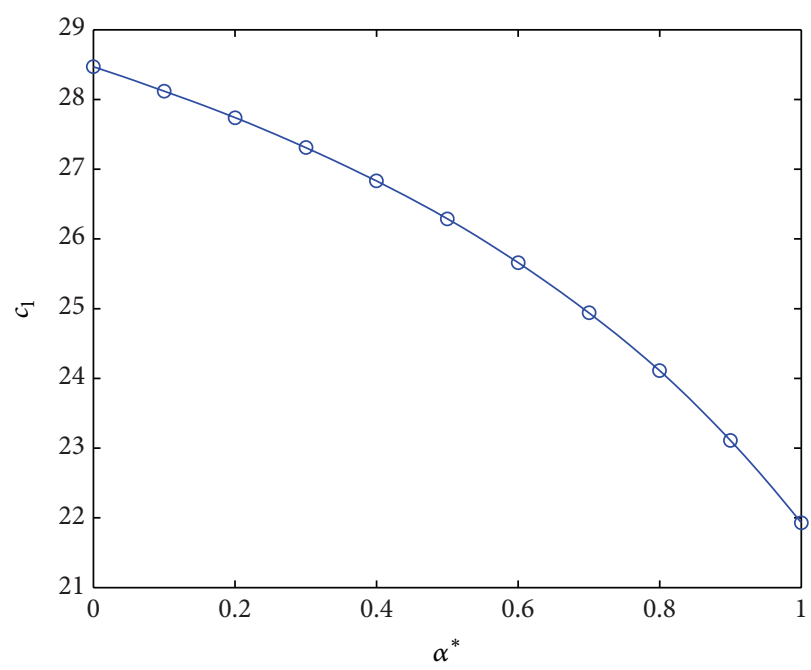

FIgURE 2: The curve of $c_{1}$ along with $\alpha^{*}$.

In this example, we randomly choose $l=\lfloor\delta N\rfloor$ nodes with a fraction $\delta=0.2$. For this E-R network with $\beta^{*}=0.4$ and given control gains $k_{1}=k_{2}=\cdots=k_{l}=0.1$, when the upper bound $c_{2}=3$ and $\widehat{c}=(1 / 2)\left(c_{1}+c_{2}\right)$, by Theorem 8 , we could get the curve of $c_{1}$ along with $\alpha^{*}$ varying in $[0,1]$. Based on Figure 2, it is known that the larger $\alpha^{*}$ results in the smaller $c_{1}$. This conclusion is consistent with constant coupling strength case that the larger coupling strength results in the easier synchronization of complex network. In order to establish the correlation between $\widehat{c}$ and $\alpha^{*}$, we select $c_{1}=20$ and $c_{2}=$ 100. Under the similar conditions and by Theorem 8 , one can have the correlation between $\widehat{c}$ and $\alpha^{*}$, which is illustrated in Figure 3. It is also concluded that the smaller $\alpha^{*}$ leads to the larger $\widehat{c}$. Moreover, when $\alpha^{*}$ is smaller, $\alpha^{*} \leq 0.2$, the value of $\widehat{c}$ changes actually and is very near to the upper bound $c_{2}$. Particularly, it is worth mentioning that when $\alpha^{*}$ is very small, $\alpha^{*} \ll 0.1$, there will be no solvable solution to $\widehat{c}$ in terms of $\widehat{c}$ being larger than its upper bound $c_{2}=100$. In this sense, it is seen that such probabilities play important roles in pinning synchronization of complex networks. Next, we will demonstrate the correlation between probabilities $\alpha^{*}$ and $\beta^{*}$. Letting $c(t) \in\left[c_{1}, c_{2}\right]$ with $c_{1}=15$ and $c_{2}=55$, a simulation of 


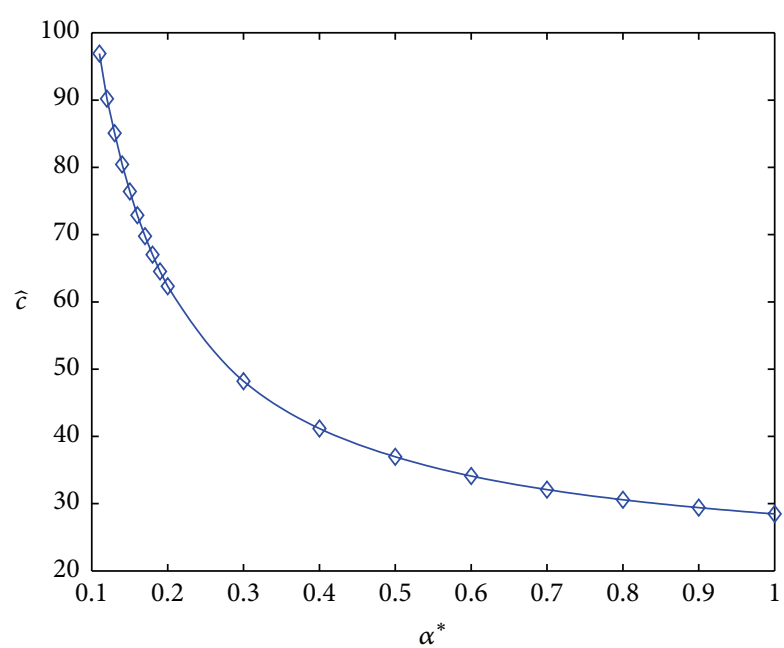

FIgURE 3: The curve of $\widehat{c}$ along with $\alpha^{*}$.

probabilities $\alpha^{*}$ and $\beta^{*}$ will be obtained by Theorem 8 , which is shown in Figure 4 . Based on Figure 4, one knows that the larger $\beta^{*}$ results in the larger $\alpha^{*}$. In other words, the larger $\beta^{*}$ means that the probability of delayed coupling taking place is larger, which needs the desired pinning controller to be added with a higher probability. When such probabilities are unavailable, we will exploit the adaptive control to realize the purpose of pinning synchronization. Without loss of generality, here it is assumed that $\beta^{*}=0.3$ is known, while $\alpha^{*}=0.2$ is inaccessible. If $\alpha^{*}$ is unknown, let the initial condition $x_{0}=$ $\left[\begin{array}{lll}-1 & 0.5 & 0.1\end{array}\right]^{T}$; by Theorem 10 , we have the state responses of synchronization error and estimation $\alpha^{*}$ shown in Figures 5 and 6. Particularly, Figure 5 is the simulation of state error $\left|e_{i}(t)\right|, i=1,2, \ldots, 10$, instead of $e_{i}(t)$. The reason is we want to demonstrate the state response of $e_{i}(t)$ in detail. Unfortunately, there are 10 nodes, where every node has 3 variables. Then, there will be 30 variables to be illustrated in one figure. This will make the state response of $e_{i}(t), i=$ $1,2, \ldots, 10$, difficult to be distinguished. However, if we plot $\left|e_{i}(t)\right|$ instead of $e_{i}(t)$, there will be 10 lines in one figure. Then, we will identify them easily. But, the effects of $\left|e_{i}(t)\right|$ and $e_{i}(t)$ implying that $e(t)$ is stable are same. All the simulations show the utility of the proposed methods in this paper.

\section{Conclusion}

In this paper, based on the stochastic viewpoint, we have investigated the pinning synchronization problem of complex networks with time-varying coupling strength and nondelayed and delayed coupling. Here, the lower and upper bounds in addition to the probability distribution of each subinterval are firstly considered in designing a pinning controller. Moreover, the nondelayed and delayed coupling here are asynchronous, whose switchings are described by a Bernoulli variable. Then, a new kind of local state feedback controller is developed without using time-varying coupling strength signal, which is dependent on such bounds and probabilities. When such probabilities are unavailable, two

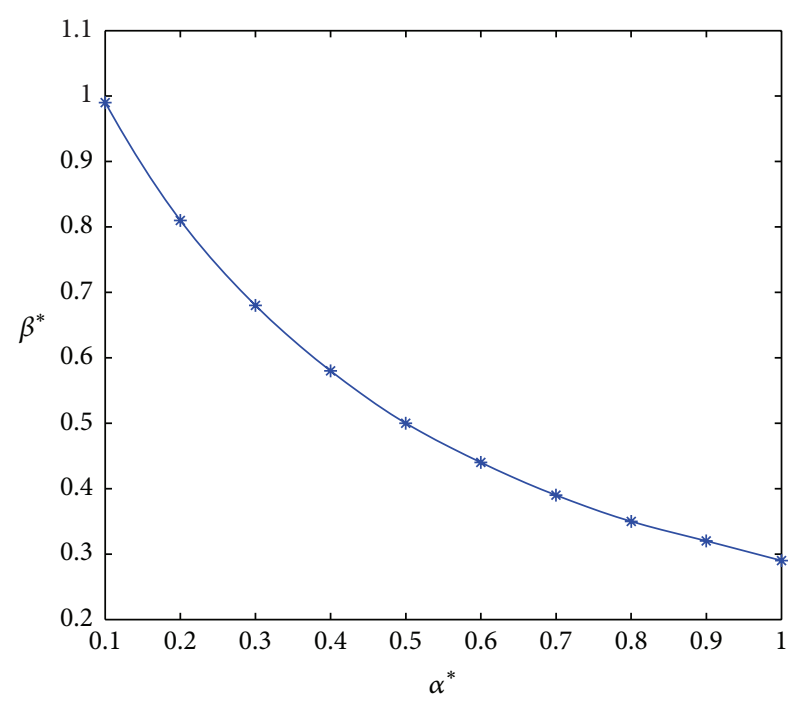

FIgURE 4: The curve of $\beta^{*}$ along with $\alpha^{*}$.

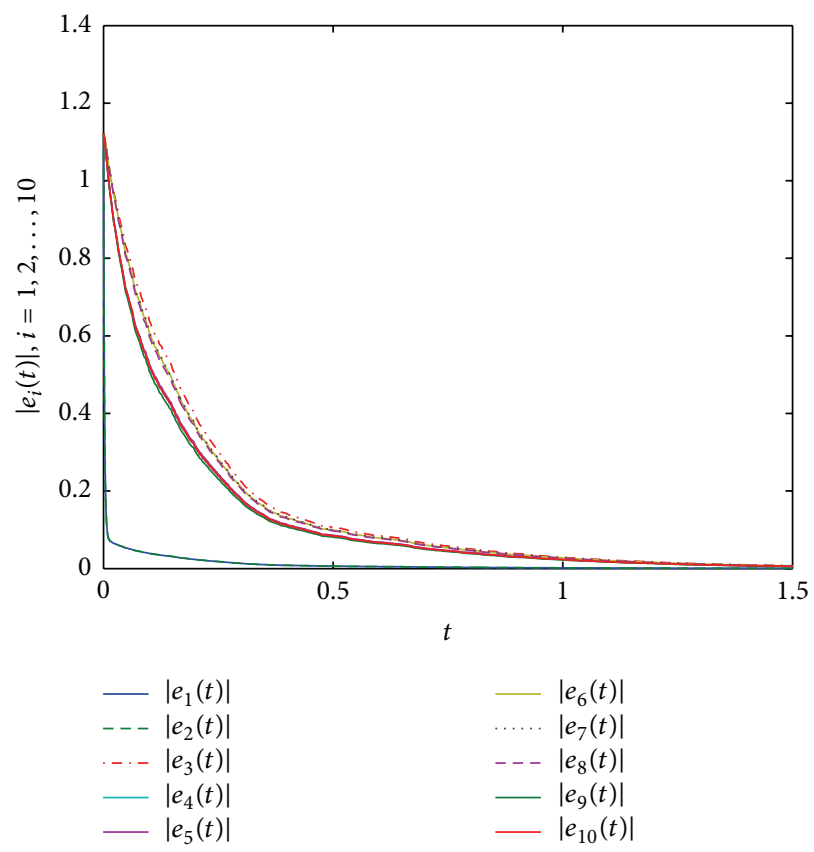

FIGURE 5: Synchronization error $\left|e_{i}(t)\right|$ by adaption controller (41).

different kinds of adaption laws are proposed to estimate the unknown parameters, respectively, and also guarantee the global synchronization of complex networks. Finally, a numerical example is given to show the utility of the presented theories. Future research could be considered to the pinning synchronization of complex networks with nondelayed and time-varying delayed couplings, stochastic pinning controller added to some nodes in term of Brownian noise, and such Bernoulli variables $\alpha(t)$ and $\beta(t)$ replaced by Markov process. 


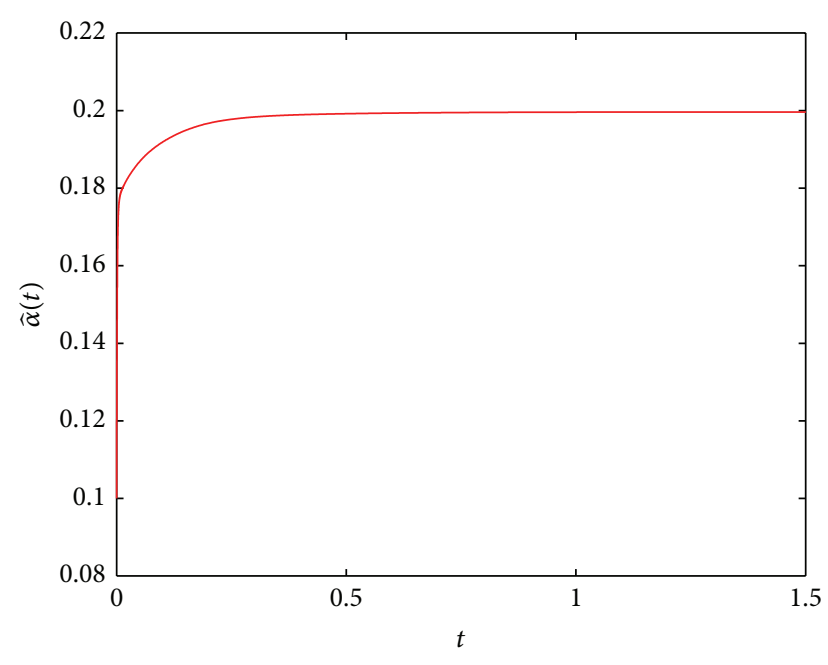

FIgURE 6: Response curve of the estimation of $\alpha^{*}$.

\section{Conflict of Interests}

The authors declare that there is no conflict of interests regarding the publication of this paper.

\section{Acknowledgments}

This work was supported by the National Natural Science Foundation of China under Grants 61104066, 61374043, and 61473140, the China Postdoctoral Science Foundation funded project under Grant 2012M521086, the Program for Liaoning Excellent Talents in University under Grant LJQ2013040, and the Natural Science Foundation of Liaoning Province under Grant 2014020106. A preliminary version of this work first appeared at the 31st Chinese Control Conference, Hefei, China.

\section{References}

[1] X. F. Wang and G. R. Chen, "Complex networks: small-world, scale-free and beyond," IEEE Transactions on Circuits and Systems-I: Regular Papers, vol. 3, no. 1, pp. 6-20, 2003.

[2] Y. Hatano and M. Mesbahi, "Agreement over random networks," IEEE Transactions on Automatic Control, vol. 50, no. 11, pp. 1867-1872, 2005.

[3] M. Porfiri and D. J. Stilwell, "Consensus seeking over random weighted directed graphs," IEEE Transactions on Automatic Control, vol. 52, no. 9, pp. 1767-1773, 2007.

[4] W. W. Yu, G. Chen, Z. Wang, and W. Yang, "Distributed consensus filtering in sensor networks," IEEE Transactions on Systems, Man, and Cybernetics, Part B: Cybernetics, vol. 39, no. 6, pp. 1568-1577, 2009.

[5] D. G. Xu and Z. F. Su, "Synchronization criterions and pinning control of general complex networks with time delay," Applied Mathematics and Computation, vol. 215, no. 4, pp. 1593-1608, 2009.

[6] H. Zhang, X. Liu, J. Wang, and H. R. Karimi, "Robust $H_{\infty}$ sliding mode control with pole placement for a fluid power electrohydraulic actuator (EHA) system," The International
Journal of Advanced Manufacturing Technology, vol. 73, no. 58, pp. 1095-1104, 2014.

[7] H. Zhang and J. M. Wang, "State estimation of discrete-time Takagi-Sugeno fuzzy systems in a network environment," IEEE Transactions on Cybernetics, 2014.

[8] P. Erdös and A. Rényi, "On the random graphs," Publicationes Mathematicae, vol. 6, pp. 290-297, 1959.

[9] D. J. Watts and S. H. Strogatz, "Collective dynamics of 'smallworld' networks,” Nature, vol. 393, no. 6684, pp. 440-442, 1998.

[10] A.-L. Barabási and R. Albert, "Emergence of scaling in random networks," Science, vol. 286, no. 5439, pp. 509-512, 1999.

[11] X. Li, X. Wang, and G. Chen, "Pinning a complex dynamical network to its equilibrium," IEEE Transactions on Circuits and Systems. I. Regular Papers, vol. 51, no. 10, pp. 2074-2087, 2004.

[12] T. P. Chen, X. W. Liu, and W. L. Lu, "Pinning complex networks by a single controller," IEEE Transactions on Circuits and Systems I: Regular Papers, vol. 54, no. 6, pp. 1317-1326, 2007.

[13] X. F. Wang and G. R. Chen, "Pinning control of scale-free dynamical networks," Physica A, vol. 310, no. 3-4, pp. 521-531, 2002.

[14] J. Lü and G. Chen, "A time-varying complex dynamical network model and its controlled synchronization criteria," IEEE Transactions on Automatic Control, vol. 50, no. 6, pp. 841-846, 2005.

[15] W. Lu and T. Chen, "New approach to synchronization analysis of linearly coupled ordinary differential systems," Physica D: Nonlinear Phenomena, vol. 213, no. 2, pp. 214-230, 2006.

[16] Y. Wang, Z. D. Wang, and J. L. Liang, "A delay fractioning approach to global synchronization of delayed complex networks with stochastic disturbances," Physics Letters A, vol. 372, no. 39, pp. 6066-6073, 2008.

[17] T. Liu, J. Zhao, and D. J. Hill, "Synchronization of complex delayed dynamical networks with nonlinearly coupled nodes," Chaos, Solitons \& Fractals, vol. 40, no. 3, pp. 1506-1519, 2009.

[18] Y. Y. Wu, W. Wei, G. Y. Li, and J. Xiang, "Pinning control of uncertain complex networks to a homogeneous orbit," IEEE Transactions on Circuits and Systems II: Express Briefs, vol. 56, no. 3, pp. 235-239, 2009.

[19] H. Li and D. Yue, "Synchronization stability of general complex dynamical networks with time-varying delays: a piecewise analysis method," Journal of Computational and Applied Mathematics, vol. 232, no. 2, pp. 149-158, 2009.

[20] Q. Song and J. Cao, "On pinning synchronization of directed and undirected complex dynamical networks," IEEE Transactions on Circuits and Systems. I. Regular Papers, vol. 57, no. 3, pp. 672-680, 2010.

[21] H. Zhang, X. J. Zhang, and J. M. Wang, "Robust gain-scheduling energy-to-peak control of vehicle lateral dynamics stabilisation,” Vehicle System Dynamics, vol. 52, no. 3, pp. 309-340, 2014.

[22] J. Zhou, J.-A. Lu, and J.-H. Lü, "Adaptive synchronization of an uncertain complex dynamical network," IEEE Transactions on Automatic Control, vol. 51, no. 4, pp. 652-656, 2006.

[23] P. DeLellis, M. diBernardo, and F. Garofalo, "Novel decentralized adaptive strategies for the synchronization of complex networks," Automatica, vol. 45, no. 5, pp. 1312-1318, 2009.

[24] W. Yu, G. Chen, and J. Cao, "Adaptive synchronization of uncertain coupled stochastic complex networks," Asian Journal of Control, vol. 13, no. 3, pp. 418-429, 2011.

[25] H. J. Gao, J. Lam, and G. R. Chen, "New criteria for synchronization stability of general complex dynamical networks with coupling delays," Physics Letters A: General, Atomic and Solid State Physics, vol. 360, no. 2, pp. 263-273, 2006. 
[26] J. Lu and D. W. Ho, "Local and global synchronization in general complex dynamical networks with delay coupling," Chaos, Solitons and Fractals, vol. 37, no. 5, pp. 1497-1510, 2008.

[27] T. Liu, J. Zhao, and D. J. Hill, "Exponential synchronization of complex delayed dynamical networks with switching topology," IEEE Transactions on Circuits and Systems. I. Regular Papers, vol. 57, no. 11, pp. 2967-2980, 2010.

[28] W. Guo, F. Austin, S. Chen, and W. Sun, "Pinning synchronization of the complex networks with non-delayed and delayed coupling," Physics Letters A, vol. 373, no. 17, pp. 1565-1572, 2009.

[29] Z. Li, L. C. Jiao, and J.-J. Lee, "Robust adaptive global synchronization of complex dynamical networks by adjusting timevarying coupling strength," Physica A: Statistical Mechanics and Its Applications, vol. 387, no. 5-6, pp. 1369-1380, 2008.

[30] H. Zhang, Y. Shi, and J. Wang, "On energy-to-peak filtering for nonuniformly sampled nonlinear systems: a markovian jump system approach," IEEE Transactions on Fuzzy Systems, vol. 22, no. 1, pp. 212-222, 2014. 


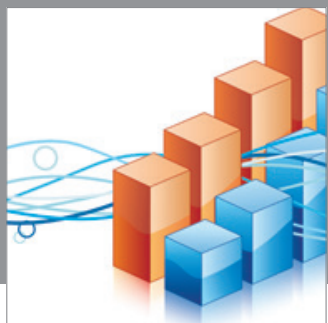

Advances in

Operations Research

mansans

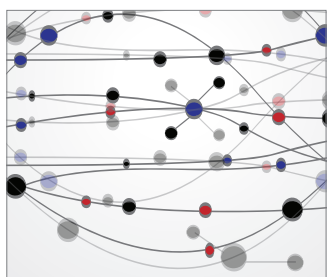

The Scientific World Journal
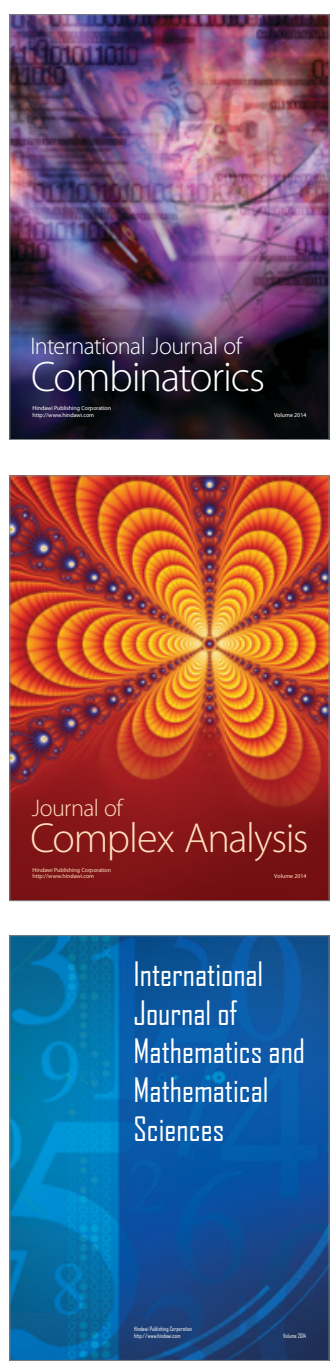
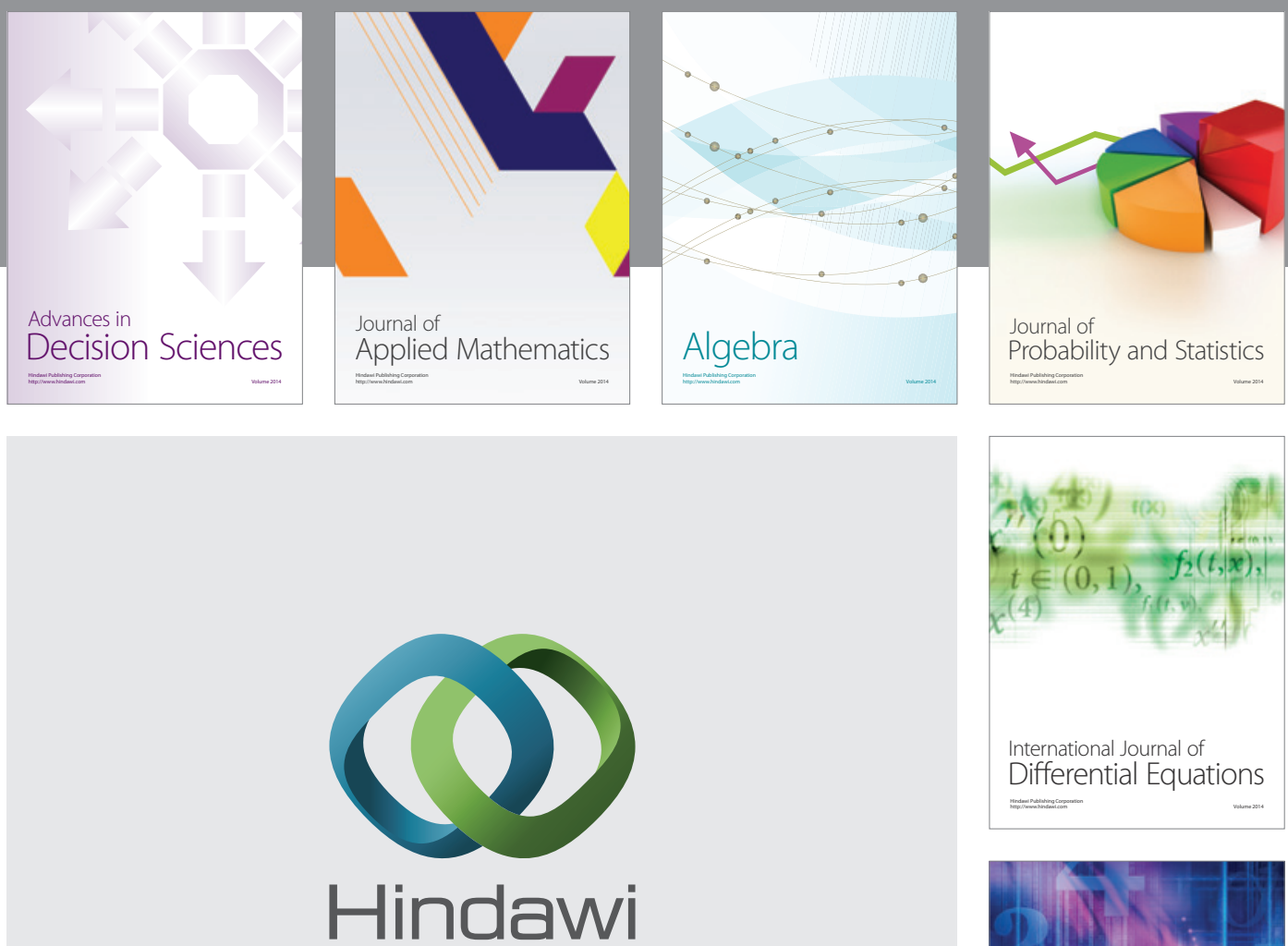

Submit your manuscripts at http://www.hindawi.com
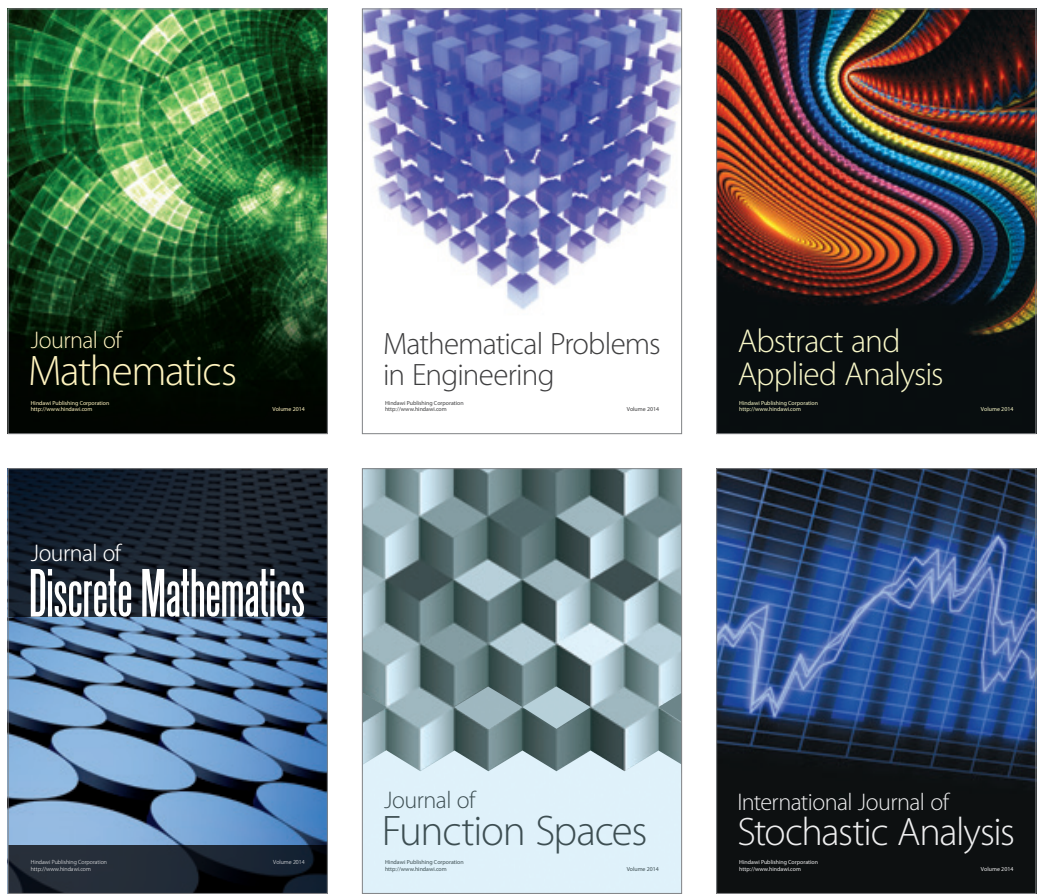

Journal of

Function Spaces

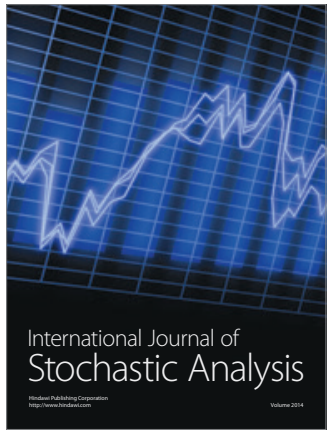

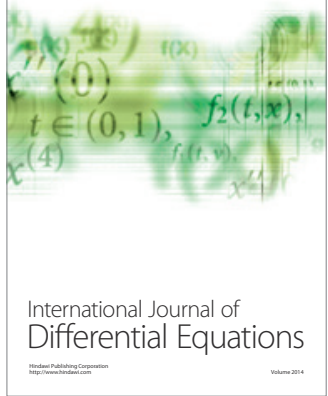
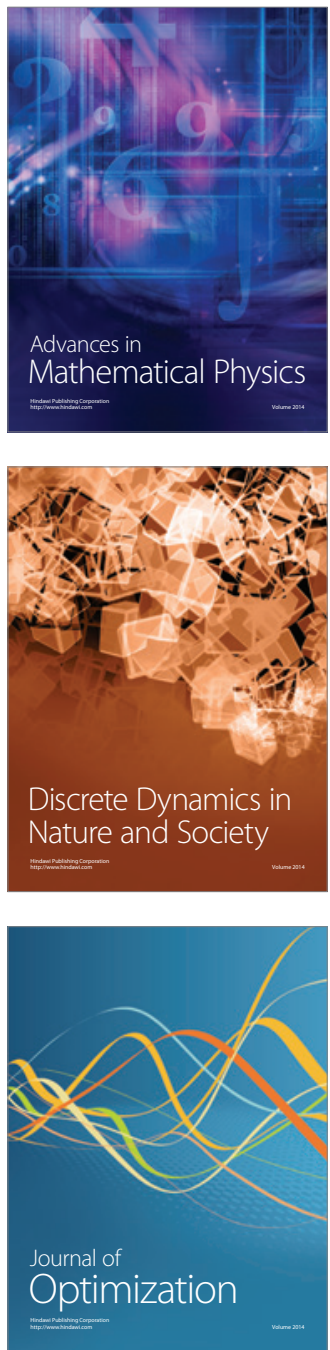